\title{
CAMBIO TECNOLÓGICO Y NUEVA SOCIEDAD DE LA INFORMACIÓN (CIBERSOCIEDAD). (CONTINUACIÓN)
}

\section{TECHNOLOGICAL CHANGE AND NEW INFORMATION SOCIETY (CYBERSOCIETY). (CONTINUED)}

\section{AUTORES}

Luis Joyanes Aguilar: Universidad Pontificia de Salamanca. Licenciado y Doctorado en Ciencias Físicas, Doctor en Informática y Doctor en Sociología.

luis.joyanes@aol.com

\section{CURRÍCULUM VITAE}

Universidad Pontificia de Salamanca. Licenciado y Doctorado en Ciencias Físicas, Doctor en Informática y Doctor en Sociología.

\section{RESUMEN}

En esta sección dedicada al mundo de la comunicación informática, cibermaneras, venimos publicando el trabajo de Luis Joyanes Aguilar, que en su segundo título denominó: CIBERSOCIEDAD. Debemos esta primicia a la gentileza de Joaquín Bandera, director de la revista: Cuadernos de Realidades Sociales, del Instituto de Sociología Aplicada de Madrid.

\section{PALABRAS CLAVE}

Tecnología - Sociedad - Información 


\section{ABSTRACT}

In this section devoted to the world of computer communication, cibermaneras, we have been publishing the work of Luis Joyanes Aguilar, who in his second title called: CyberSociety. We owe this to the kindness scoop Flag Joaquin, director of the journal: Journal of Social Realities, Institute of Applied Sociology Madrid.

\section{KEY WORDS}

Technology - Society - Information

\section{ÍNDICE}

Cambio tecnológico y nueva sociedad de la información (CIBERSOCIEDAD). Por Luis Joyanes Aguilar. Cuarta parte: Las nuevas tecnologías

9. Las innovaciones tecnológicas en la información NOTAS.

En esta sección dedicada al mundo de la comunicación informática, "cibermaneras", venimos publicando el trabajo de Luis Joyanes Aguilar, que en su segundo título denominó "CIBERSOCIEDAD". Debemos esta primicia a la gentileza de Joaquín Bandera, director de la revista "Cuadernos de Realidades Sociales", del Instituto de Sociología Aplicada de Madrid.

La extensión de este texto, realmente titulado "Cambio tecnológico y nueva sociedad de la información", hizo inviable su publicación en un sólo número de Vivat 
Academia, razón por la cual hemos decidido ofrecerlo a nuestros lectores en varias entregas; aquí tenemos la Cuarta.

Esta vez, tras definir lo que podemos entender por "Cambio Tecnológico", Luis Joyanes analiza las reacciones de la sociedad actual ante las innovaciones en materia de información.

\section{LAS INNOVACIONES TECNOLÓGICAS EN LA INFORMACIÓN}

Los indicadores o valores que fundamentan la aceptación de las Nuevas Tecnologías de la Información se sintetizan bien en torno a las ideas de progreso, en general, por un lado, y de eficacia, específicamente empresarial por otro. Otros autores consideran como indicadores los factores de eficiencia, que se definen como el cociente entre la salida y entrada a un sistema económico [(bienes y servicios)/recursos].

¿Qué se puede considerar como cambio tecnológico? Galán González considera que toda modificación del funcionamiento de un sistema que suponga una transformación del indicador de eficiencia se considera como cambio tecnológico. Sin embargo, no se puede considerar un cambio tecnológico aquel que provoque una disminución del índice de eficiencia, al menos permanentemente. Smith considera que: "el cambio tecnológico se acompaña de un incremento de la producción y de la productividad de los medios de producción"Nota43. Existe, no obstante, disparidad en cuanto a cuáles son los indicadores que afectan al cambio tecnológico. Así, BlattnerNota44 señala:

"el cambio tecnológico no necesariamente ha de ser equivalente a un progreso técnico, que es aquella parte del cambio tecnológico que conduce a un incremento en la productividad total de los factores o a la reducción de los costes marginales de producción, al menos en alguna parte de la economía". 
Esta hipótesis reconoce que existe posibilidad de cambio tecnológico sin progreso técnico, es decir, sin incremento de la eficiencia. De cualquier manera, como ya aventuraba una de las conclusiones del informe "FAST. Europa 1995", la innovación tecnológica, no garantiza por sí misma ningún tipo de cambio económico, social o político, ya que los efectos reales y, sobre todo, la bondad o maldad de cualquier nueva tecnología o de todas las nuevas tecnologías en conjunto, depende de quién las implante y cómo se controle y oriente esta implantación. Se destaca la importancia que tienen las denominadas "políticas de modernización", verdaderos planes más o menos concertados entre los diferentes actores económicos, sindicales y políticosNOTA45.

El desarrollo e implantación de las nuevas tecnologías afecta tanto a los procesos de producción e intercambio económico como al ritmo de transformación de la sociedad, que a su vez, actúa sobre la estructura ocupacional, la demografía, la educación y la familia. Y en un escalón superior, operaría sobre la forma y composición de la estratificación social, así como sobre la dinámica de las clases.

La forma, estructura y contenido del sistema social del futuro será bastante diferente del actual, del de la sociedad industrial y posindustrial. Es sin ninguna duda, el carácter comunicativo e informativo de muchas de las nuevas tecnologías y su poder de transformación uno de los rasgos definidores de la "nueva sociedad de la información"NOTA46. La revolución tecnológica que supone la sociedad de la información se basa en que la materia prima es la información, al contrario, la primera y segunda revolución industrial tenían como fundamento la máquina de vapor y la electricidad. 
Expectativas de la nueva sociedad de la información

Las estructuras que ha creado la Sociedad de la Información se han articulado alrededor de ciertos aspectos ideales o utópicos que CastillaNOTA47 en 1987 ya sintetizaba en las siguientes, que siguen siendo válidas hoy día:

- Supresión/superación de la crisis.

- Acabar con el paro y transformarlo en ocio.

- El tiempo libre para mejorar el bienestar.

- Mejora del ecosistema.

Los sectores más proclives a la aceptación de las Tecnologías de la Información

Aunque en estos últimos años la mentalidad moderna ha cambiado radicalmente, todavía es posible encontrar sectores, personas o instancias más próximas o lejanas a la aceptación de las nuevas tecnologías de la información. Los dos sectores que todos los grandes especialistas de la sociedad de la información consideran más proclives al advenimiento de la misma son:

- Los jóvenes y los niños (aspecto generacional)

- Los empresarios (aspecto económico)

El niño que se encuentra estudiando primaria y los jóvenes que estudian bachiller y formación profesional son las personas más proclives a comprender las grandes ventajas que entraña la sociedad de la información y, sobre todo, a comprender el cambio social y los impactos que él mismo producirá. El otro sector proclive a la sociedad de la información y a incorporar las nuevas tecnologías, son los empresarios 
y las empresas en general. La eficacia es la motivación de su total proclividadNOTA48 ya que esperan resultados tales como:

- mayor rendimiento/productividad

- menor costo

- menos personal

- mejor información

- rapidez en la decisión

En líneas generales, CastillaNOTA49 señala que el perfil de adopción de las nuevas tecnologías de la información por parte de la empresa se podría dividir de acuerdo a estos grupos y características:

a) Empresa grande

- Todos los servicios

- Actitud muy positiva

- Consideración necesaria

- Introducción rápida

b) Empresa mediana

- Servicios informáticos casi exclusivamente

- Problemas de no ser/verle rentabilidad 
c) Empresa pequeña

- En los sectores más dinámicos y en las empresas familiares tradicionales pero con directivos jóvenes: la única preocupación es el temor a equivocarse en la compra e inversiones necesarias

- En las empresas de corte más tradicional y con directivos de elevada edad

- Resistencia por cuestiones de "mentalidad"; más propensos a invertir en valores tradicionales que en tecnologías modernas. Problemas de costos.

Las grandes empresas, como los Bancos, industrias del automóvil, de telecomunicaciones, etc., son las más propensas a la informatización, y uno de sus grandes argumentos es el tipo de formación de la mayoría de sus ejecutivos, entre los que tienen formaciones gradualmente afines con la información: informáticos, graduados de ciencias e ingeniería, ejecutivos, etc.

Resistencia o rechazo antes las Tecnologías de la Información y las Comunicaciones

Son numerosos los aspectos de resistencia/rechazo implicados en las innovaciones tecnológicas en información, aunque la postura final termina ya siendo globalmente de aceptación. CastillaNOTA50 considera que los factores principales de aceptaciónrechazo/resistencia son:

- Aceptación: eficacia y progreso

- Resistencia/rechazo: temor al cambio

Las encuestas más fiables realizadas en los últimos diez años muestran que el temor al cambio $\mathrm{y}$ a sus efectos son -sin género de dudas- el factor de mayor resistencia/rechazo a las Tecnologías de la Información. Sin embargo, estamos de acuerdo -en el año 1996- con la teoría de Castilla -del año 1987- de que la aplicación 
masiva de las Tecnologías de la Información implica en realidad la implantación definitiva de la "Sociedad de la Información" y es: "por encima de toda cosa, el cambio, el cambio de todo orden: cambio de vida, de conceptos de muchas cosas, la pérdida de los esquemas fijos, etc."NOTA51.

Sin embargo queremos considerar una de las "amenazas" que más temen las personas en las industrias, los negocios y el mundo de la educación: "el poder de la información". Los empleados temen, pensando en el futuro, no "poseer en exclusiva" la información necesaria para la vida diaria y para la planificación del futuro; así mismo, se tiene el temor a la pérdida del protagonismo individual o al debilitamiento de las características tradicionales de los roles profesionales (poder, jerarquía, etc). Es lo que llaman los sociólogos la igualación social, término sobre el que volveremos más adelante cuando nos introduzcamos en el cuerpo de la investigación.

Aspectos y actitudes negativas de las personas

Las repercusiones negativas en las personas vienen dadas porque las mismas no sólo afectan al individuo en sí, sino que su desarrollo alcanza a las esferas de las relaciones sociales, laborales, políticas y -fundamentalmente- al mundo familiar. Consideremos algunas de las repercusiones negativas que se plantean en la mayoría de los casos, como aspectos míticos. Los más significativos son:

La deshumanización

Rechazo a la máquina en sus más diversos aspectos (el mítico temor a la sustitución del trabajador por la máquina aumenta cada día, sobre todo en personas con formación débil). 
El lenguaje informático. La falta de un desarrollo cultural paralelo en igual porcentaje que el desarrollo del mundo de la información hace que el desconocimiento y la no comprensión del leguaje informático impongan una barrera difícil de salvar.

El trabajo en casa. Debido, fundamentalmente, al aumento imparable del teletrabajo, comienza a pesar como una losa en los "enemigos" de esta modalidad de trabajo. El hogar aparece amenazado y la sociedad interconectada de James MartínNOTA52 hace que dicho hogar pierda su figura tradicional y comienza a considerarse como una extensión del lugar de trabajo, en detrimento de la preponderancia total que siempre ha tenido la familia.

La intimidad y el peligro a su pérdida es otro de los aspectos casi negativos que las personas más temen ante el avance de las Tecnologías de la información.

El colonialismo tecnológico. La dependencia extranjera en tecnología punta suele ser motivo de preocupación social, especialmente en el mundo empresarial y de negocios.

El sentimiento de sentirse controlado. Las Tecnologías de la Información, sobre todo desde la implantación de las grandes redes de ordenadores y las autopistas de la información, han impuesto el sentimiento, que en muchos casos es convicción, de sentirse controlados por el Gran Hermano de Orwell. Pensemos en el caso de los grandes ordenadores de Hacienda que cada día tienen más controlado al contribuyente.

Aunque en el año 2000 no se puede hablar de rechazo o resistencia generalizada, ni incluso parcial, sí es cierto que todavía existe una serie de sectores -más bien personas físicas- que sienten mayor rechazo o resistencia al advenimiento de la sociedad de la información. Este sector de personas suele ser aquel que 
sociológicamente se encuentra menos informado o aquellos que pertenecen a profesiones cuyo grado de información es pequeño y se sienten o se van a sentir "desplazados" de su actividad laboral. Destacaremos entre ellos los siguientes sectores de personas:

Administrativos: especialmente los de mayor edad y los no cualificados.

Sectores marginales. Jóvenes "sensibilizados", "ecologistas", "antinucleares", "antipolíticos", etc que ven en la Sociedad de la Información un peligro contra los principios naturales de la Humanidad.

Personas desplazadas por su formación tradicional o la singularidad de su puesto de trabajo.

Intelectuales, como prototipos de contestación antitecnológica por lo que representa la pérdida de la creación personal en favor de la creación intelectual basada en el ordenador

Pequeños y medianos empresarios. Esencialmente motivados por una falta de "mentalización" y normalmente con problemas de inversión.

CONTINUARÁ...

En el próximo número:

SOCIEDAD E INFORMACIÓN 


\section{NOTAS}

NOTA 43: J. S. SMITH, citado por José Luis Galán González en op. cit., pág. 271.

NOTA 44: N. BLATTNER, "Labour Displacement by Technological Change? A preliminary survey of the case of microelectronics", en Revista Internazionale di Science Economiche e Commerciali no 5, Mayo 1981, pág. 424.

NOTA 45: Informe FAST. Europa 1995. Comisión de las Comunidades Europeas. Madrid, Fundesco, 1986.

NOTA 46: MIGUEL ROIZ, Nuevas tecnologías y transformación de la estructura social española en Documentación Social, Cáritas Española, Madrid, 1986, nº 65, pág. 35 .

NOTA 47: ADOLFO CASTILLA, M ${ }^{a}$ CRUZ ALONSO Y JOSÉ ANTONIO DÍAZ, La Sociedad española ante las nuevas tecnologías, Madrid, Fundesco, 1987. págs. 84-86.

NOTA 48: Ibíd., pág. 92.

NOTA 49: Ibíd., pág. 93.

NOTA 50: Ibíd., págs. 93-94.

NOTA 51: Ibíd., pág. 95.

NOTA 52: James Martin es un experto mundial en tecnologías de la información. Sus previsiones de futuro tecnológico se suelen cumplir casi siempre. Su obra "La sociedad interconectada", Madrid, Tecnos, 1978, cobra hoy vigencia dado que la 
sociedad interconectada que predijo, hoy es la sociedad de la información, con la mayoría de todas sus previsiones cumplidas.

Con anterioridad a James Martin, y en los conflictivos años 1967/68 que desde el punto de vista científico fueron transcendentales, dos científicos americanos Herman Kahn y Anthony J. Wiener, miembros del Hudson Institute de EEUU, publicaron un estudio que adquirió gran relevancia científico-social sobre las 100 innovaciones tecnológicas más probables que se producirían en el último tercio del siglo XX; de ellas muchas son relativas a Tecnologías de la Información. Su obra The Year Two Thousands, New York, McMillan Company, 1967, Tabla XVIII; págs. 51-55 fue prologada por Daniel Bell, por aquel entonces presidente de la American Academic of Arts and Sciences de EEUU. Es de destacar también la obra Hacia el año 2000. Los próximos años de H. Kahn y E. Rostow et al, Barcelona, Kairos, 1967 que trataba sobre los futuros alternativos del mundo. 